\title{
Biens Symboliques / Symbolic Goods
}

Revue de sciences sociales sur les arts, la culture et les idées

\section{D'un champ littéraire introuvable aux dispositions et pratiques des écrivain'e's tunisien'ne's}

From an Untraceable Literary Field to the Dispositions and Practices of Tunisian Writers

De un campo literario inencontrable a disposiciones y prácticas de escritor $\cdot e \cdot a \cdot s$

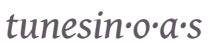

\section{Abir Kréfa}

\section{(2) OpenEdition}

Journals

Édition électronique

URL : http://journals.openedition.org/bssg/137

DOI : $10.4000 /$ bssg. 137

ISSN : 2490-9424

Éditeur

Presses universitaires de Vincennes

Référence électronique

Abir Kréfa, «D'un champ littéraire introuvable aux dispositions et pratiques des écrivain.e.s

tunisien.ne.s », Biens Symboliques / Symbolic Goods [En ligne], 1 | 2017, mis en ligne le 15 octobre 2017, consulté le 04 mars 2021. URL : http://journals.openedition.org/bssg/137 ; DOI : https://doi.org/ $10.4000 /$ bssg. 137 

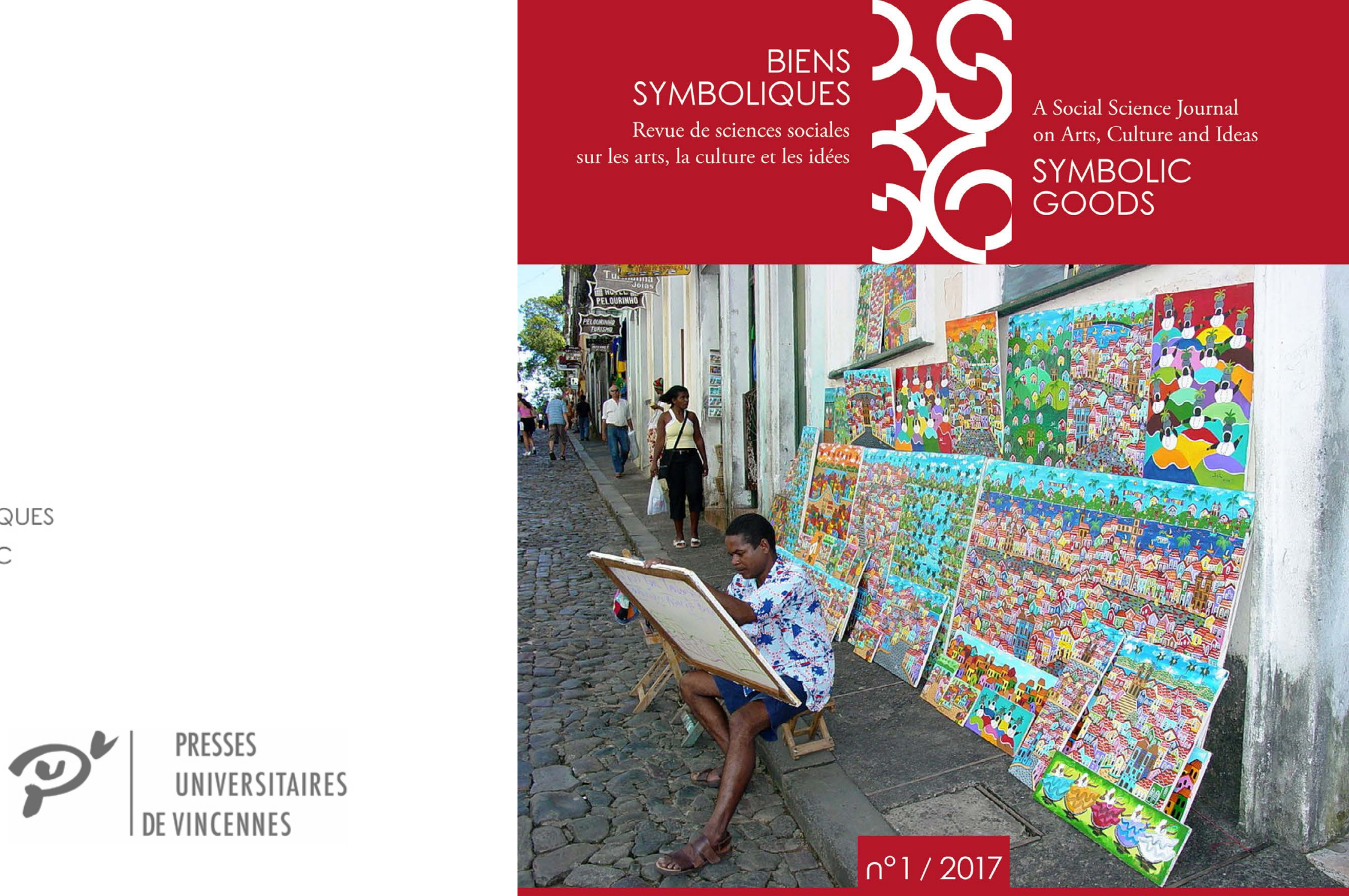

\section{Artistes ordinaires \\ Ordinary Artists}




\title{
D'un champ littéraire introuvable aux dispositions et pratiques des écrivain'e-s tunisien-ne's
}

\author{
Abir Kréfa
}

Conçu pour analyser les pratiques culturelles en milieux populaires, l'ouvrage de Claude Grignon et Jean-Claude Passeron (1989) est en fait heuristique pour appréhender, au-delà de cet exemple, les univers culturels ou symboliques peu reconnus. Quand j'ai commencé en 2006 ma thèse de sociologie sur les rapports de genre dans l'univers littéraire tunisien (Kréfa 2013), j'étais fortement imprégnée des travaux de Pierre Bourdieu. Mon projet visait, sur le modèle des Règles de l'art (Bourdieu 1992), à effectuer une cartographie du « champ » littéraire tunisien, en reconstituant ses différents pôles, tout en y introduisant la dimension du genre. Cependant, dans la mesure où les concepts de la sociologie conservent souvent une référence tacite à des coordonnées spatio-temporelles (Passeron 1991), ils ne peuvent être mécaniquement transposés à d'autres réalités socio-historiques. Discuté pour le cas français (Lahire 2012), le concept de “champ » risque de verser sans cesse dans le misérabilisme pour ne qualifier les réalités littéraires tunisiennes que par défaut, sans les analyser ni les expliquer. Tout au plus pourrait-on décliner les « causes » de «l'inexistence » d'un « champ » : un « petit » pays, de dix millions d'habitants environ, où l'alphabétisation est « récente » et le prix du livre relativement élevé ; la faible canonisation de la littérature tunisienne par l'institution scolaire ; le contrôle des activités littéraires en contexte autoritaire ; la dépendance de cet univers aux champs littéraires centraux (Casanova 2008), etc.

L'œuvre de Claude Grignon et de Jean-Claude Passeron, mais aussi les travaux sur le genre en art et en littérature (Buscatto 2014 ; Dumont \& Sofio 2007 ; Naudier \& Rollet 2007), m'ont conduite à opérer des déplacements dans la construction de l'objet. La première rupture a consisté à prêter attention à ce que font les écrivain·e.s (plutôt qu'à ce qu'ils et elles sont supposé.e.s faire) et donc à prendre au sérieux leurs activités littéraires. L'objectivation des activités littéraires invitant à reconstituer les dispositions qui y inclinent, j'ai pris pour objet la genèse des pratiques d'écriture, les contextes (Lahire 2012) et les événements biographiques déclencheurs, ainsi que les instances de socialisation (familiale, scolaire, groupe de pairs, etc.) ayant favorisé l'acquisition des compétences littéraires. Reconstituer celles-ci le plus finement possible amène à relativiser certaines oppositions, comme entre écritures littéraires et non littéraires, ou entre oralité et écriture. Par ailleurs, introduire le genre conduit à se distancier d'une conception institutionnelle de la censure et d'une définition légitimiste des instances de réception des œuvres. Le dernier déplacement a porté sur les rapports entretenus par les écrivain·e.s à la « légitimité culturelle ». 


\section{Dits et écrits « ordinaires », « scolaires » et «littéraires »}

À rebours de tout légitimisme, les écritures « ordinaires» (telles les correspondances, les journaux intimes, les lettres d'amour, les carnets de notes, etc.) ont été prises en considération pour analyser la genèse des pratiques et des dispositions littéraires. Tout en étant « produites par des gens ordinaires, sans titre ni qualité » (Chartier 2001), les écritures ordinaires peuvent en effet être investies esthétiquement : ceux et celles qui écrivent, pour soi ou pour d'autres, peuvent chercher à s'exprimer avec « élégance », à accorder de l'attention à la forme et au style, aux manières de dire et pas uniquement au contenu de ce qui est dit. Comme le fait remarquer Claude Fossé-Poliak (2006 : 169), les « écritures ordinaires peuvent [au final] accompagner des pratiques plus littéraires ou y prédisposer ». De la même manière, les différences entre « écritures scolaires » des disciplines littéraires et « écritures littéraires » ont été relativisées. Tout en ayant une finalité instrumentale, les exercices scolaires favorisent l'acquisition des dispositions et des pratiques littéraires : d'une part parce que ceux et celles qui en sont

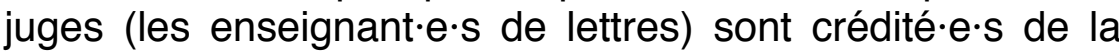
connaissance des « grands » textes littéraires, d'autre part parce que l'écriture peut prendre pour modèles des œuvres reconnues, etc.

La forte proportion d'écrivain·e.s originaires des milieux populaires parmi les enquêté.e.s arabophones invitait enfin à déconstruire l'opposition entre « oralité » et « écriture ». La plupart avaient en effet acquis le goût de la langue arabe, pour la fiction et les procédés de narration à travers leur mémorisation et récitation du Coran au kuttâb $b^{1}$, ainsi que de l'audition de contes et légendes. Pour les écrivain'e-s de langue arabe né·e·s dans un milieu familial où l'imprimé était rare, l'apprentissage du Coran a constitué un mode d'imprégnation diffus avec les structures et le vocabulaire de la langue lettrée, avant que celle-ci ne fasse l'objet d'un apprentissage écrit à partir de l'école primaire. La fréquentation du kuttâb a aussi placé les écrivain'e·s des classes populaires dans des contextes assez proches des institutions scolaires, les amenant à acquérir des dispositions valorisées par l'école : le respect de l'autorité liée à la maîtrise du langage et à la détention du savoir, un certain rapport au temps, la ponctualité et l'assiduité. À travers l'écoute de « récits » et de « monologues » puisés dans le patrimoine populaire et/ou l'histoire des premiers temps de l'islam, ces écrivain.e.s ont par ailleurs acquis une compétence à vivre le langage comme un univers autonome, indépendant des interactions et du contexte de son énonciation (Lahire 2008).

\section{Des censures et des instances de réception disséminées}

Un autre déplacement a consisté à redéfinir les instances chargées du contrôle et de la réception des œuvres. Alors que

1 Le kuttâb désigne le lieu d'apprentissage oral et collectif du Coran par les enfants. II peut s'agir d'une pièce réservée à cette fin à la mosquée ou, à proprement parler, d'une école coranique [madrassa qur'âniyya] construite par un notable, un « bienfaiteur », etc. Si la fréquentation du kuttâb est gratuite à la mosquée, une somme - le plus souvent modique - peut être exigée à l'école coranique. 
l'objectif de la thèse consistait au début à saisir les modalités de la censure étatique en contexte autoritaire ainsi que de la réception par les instances de consécration (éditeurs, critiques, pairs, etc.), il est apparu que ces définitions étaient restrictives et androcentrées. Tandis que les écrivains ne sont confrontés qu'au contrôle de leur production par les institutions étatiques - lequel s'exerçait principalement au travers du dépôt légal (Kréfa 2013) -, les écrivaines font face à une censure disséminée, très forte dans la sphère familiale et conjugale. Celle-ci prend des formes diverses, à différents moments du processus de fabrication de l'œuvre : injonctions au silence dans le cours de l'écriture, réceptions gênées ou moralisatrices, notamment quand les écrits traitent de thèmes contrevenant aux normes sociales dominantes, etc.

Ces contraintes conduisent les écrivaines à se préoccuper tout autant - sinon davantage - de la réception de leurs écrits par leur entourage, des réactions du conjoint (pour celles qui sont en couple) et des membres de la famille, que de la réception par les pairs, les critiques et les éditeurs. La nécessité de réinscrire parallèlement les œuvres dans les conditions privées de leur production et de leur réception s'est imposée de manière d'autant plus évidente que, contrairement à la censure étatique qui a fait l'objet d'une mobilisation collective d'écrivains, la censure privée demeure invisible. Elle conduit pourtant chaque écrivaine à des accommodements et à des «identités stratégiques » (Collovald 1988), renforçant ainsi la fragilité d'une identité d'auteur qui ne peut s'appuyer sur un statut professionnel (Heinich 2000). L'opération n'est cependant pas additive : il ne s'agit pas d'ajouter des instances de contrôle ou de réception à celles qui sont prises classiquement pour objet (Bourdieu 1998 ; Dragomir 2011).
Les analyser conjointement a permis de mettre en évidence, dans le cas de l'univers littéraire tunisien, que les critères d'appréciation d'une « bonne œuvre » pour les instances reconnues de consécration, ne sont pas neutres du point de vue du genre : alors que les critiques, les pairs et les éditeurs valorisent la «transgression » des tabous sexuels et religieux, celle-ci ne peut être opérée par les écrivaines sans heurter, dans la sphère familiale et conjugale, les gardiens du patronyme (Kréfa 2014).

\section{Des rapports diversifiés à la « légitimité culturelle»}

La dernière forme de distance à l'endroit du légitimisme a été de ne pas réduire les pratiques, les goûts et les aspirations des écrivain'e.s tunisien'ne-s à des goûts et des pratiques de « nécessité », pour mettre en évidence les « différences », les « variations » et les « oppositions » internes (Grignon \& Passeron 1989, p. 115) au sein d'un univers littéraire dominé. La domination littéraire exercée par les « champs littéraires » centraux (Le Caire et Beyrouth pour les arabophones, Paris pour l'ensemble des écrivains) ne se traduit pas par une homogénéisation des pratiques et des aspirations des

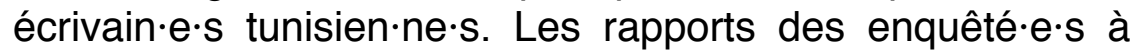
la domination littéraire ont été appréhendés à travers les modes (compte d'éditeur, compte d'auteur ou autoédition) et les lieux d'édition (en Tunisie ou à l'étranger). Une ligne de différenciation sépare les écrivain·e.s selon leur langue d'écriture. Ayant beaucoup plus nettement intériorisé la nécessaire division du travail entre l'auteur et l'éditeur, les écrivain'e.s francophones se tiennent ainsi à distance de 
l'autoédition et considèrent que seule l'édition à compte d'éditeur constitue le gage de la « qualité » de leurs œuvres, alors que cela est moins systématique chez les arabophones, dont beaucoup utilisent indifféremment les termes de « nachr » [édition] et de « tab' « [impression]. Les pratiques et les aspirations varient aussi fortement en fonction des atouts littéraires, de l'âge de la première publication, de leurs réseaux de sociabilité, rejoignant les résultats établis pour d'autres écrivain'e.s peu reconnu·e·s (Bois 2008). Au final, l'univers littéraire tunisien apparaît nettement dominé par les « centres littéraires » (Casanova 2008) que sont Paris et Le Caire, dont les structures et les œuvres constituent une référence, voire un « modèle » pour ses membres. Dans le même temps extrêmement hétérogène et éclaté, il n'est pas régi par des principes de hiérarchisation stabilisés.

Abir Kréfa Université Lyon 2/CNRS UMR 5283, Centre Max Weber https://www.centre-max-weber.fr/

\section{Références bibliographiques}

Aron Raymond (1983). Mémoires. Paris, Julliard.

BAKHTINE Mikhaïl (1970). L'CEuvre de François Rabelais et la culture populaire au Moyen Âge et sous la Renaissance. Paris, Gallimard.

BECKER Howard (1988). Les Mondes de l'art. Traduit de l'anglais par Jeanne Bouniort. Paris, Flammarion.

Bellavance Guy, Boivin Micheline, SanterRe Lise (dir.) (2000).

Démocratisation de la culture ou démocratie culturelle? Deux logiques d'action publique. Québec, Éditions de I'IQRC.

Bols Géraldine (2008). "Le consentement à la domination littéraire. Degrés et diversité de ses formes chez les écrivains "les moins reconnus" de l'univers littéraire ». Tracés, $14: 55-76$.

BOURDIEU Pierre (1979). La Distinction. Critique sociale du jugement. Paris, Minuit.
BouRdieu Pierre (1984). Questions de sociologie. Paris, Minuit.

BOURDIEU Pierre (1988). « Entretien recueilli par Beate Krais ». In BouRdiEU Pierre, Chamboredon Jean-Claude, PASSERON Jean-Claude, Le Métier de sociologue. Préalables épistémologiques. Paris, Éditions de l'EHESS (cinquième édition).

BOURDIEU Pierre (1989). La Noblesse d'État. Paris, Minuit.

BOURDIEU Pierre (1993). «Les contradictions de l'héritage ». In Bourdieu Pierre (dir.), La Misère du monde. Paris, Seuil : 711-718.

BOURDIEU Pierre (1998) [1992]. Les Règles de l'art. Genèse et structure du champ littéraire. Paris, Seuil.

BOURDIEU Pierre (1998). La Domination masculine. Paris, Seuil.

BouRdieu Pierre (2003) [1997]. Méditations pascaliennes. Paris, Seuil.

Bourdieu Pierre, Chamboredon Jean-Claude, PASSERON Jean-Claude (1968). Le Métier de sociologue. Paris, Mouton.

Bourdieu Pierre \& PASseron Jean-Claude (1964). Les Héritiers. Les étudiants et la culture. Paris, Minuit.

BouRdieU Pierre \& PASSERON Jean-Claude (1970). La Reproduction. Éléments pour une théorie du système d'enseignement. Paris, Minuit.

BOURDIEU Pierre \& WACQUANT Loïc (2014). Invitation à la sociologie réflexive. Paris, Seuil.

BOUTIER Jean (2008). "Quelques réflexions rétrospectives sur les histoires de la "culture populaire" ». Journée d'étude "Le Savant et le Populaire, 1989-2008. Retour sur un débat en suspens ». SHADYC, EHESS Marseille (non publié).

BOUVERESSE Jacques (2008). « Le besoin de croyance et le besoin de vérité ». Agone, 38-39: 281-306.

BuRkE Peter (1978). Popular Culture in Early Modern Europe. Londres, Temple Smith.

BuscatTo Marie (2014). Sociologies du genre. Paris, Armand Colin

BusıNo Giovanni (2006). «Entretien avec Dominique Schnapper ». Revue européenne des sciences sociales, XLIV(135). [En ligne] http://ress. revues.org/271 [consulté le 12 octobre 2016].

Casanova Pascale (2008) [1999]. La République mondiale des lettres. Paris, Seuil.

CHAPOULIE Jean-Michel (1991). « La seconde fondation de la sociologie française, les États-Unis et la classe ouvrière ». Revue française de sociologie $32(3)$ : 321-364 
CHARTIER Roger (2001). "Culture écrite et littérature à l'âge moderne ". Annales. Histoire, Sciences sociales, 56(4) : 783-802.

COHEN Déborah (2010). La Nature du peuple. Les formes de l'imaginaire social (XVIII $/ X X P^{e}$ siècles). Seyssel, Champ Vallon.

ColleCTIF (1988). «Histoire et sciences sociales. Un tournant critique ? 》. Annales ESC, 2 : 291-293.

Collectif RéVoltes logiques (1984). L'Empire du sociologue. Paris, La Découverte.

Collovald Annie (1988). «Identité(s) stratégiques(s) », Actes de la recherche en sciences sociales, $73: 29-40$.

Connell Raewyn (1995). Masculinities. Cambridge, Polity Press.

Cornu Tanguy (2008). « L'ambiguïté du concept de domination symbolique dans Le Savant et le Populaire ". Journée d'étude "Le Savant et le Populaire, 1989-2008. Retour sur un débat en suspens ". SHADYC, EHESS Marseille (non publié).

CoULANGEON Philippe \& DUVAL Julien (2013). Trente ans après La Distinction de Pierre Bourdieu. Paris, La Découverte.

COULANGEON, Philippe \& DUVAL Julien (2013). «Introduction ». In EID. (dir.), Trente ans après La Distinction de Pierre Bourdieu. Paris, La Découverte. DAUDET Alphonse (1868). Le Petit Chose. Paris, Pierre-Jules Hetzel.

Debeauvais Michel (1976). L'Université ouverte : les dossiers de Vincennes.

Grenoble, Presses Universitaires de Grenoble.

Dragomir Lucia (2011). "L'Union des écrivains. Un modèle institutionnel et ses limites ». Vingtième siècle, $109: 59-70$.

DUCOURNAU Claire (2017). " Les deux (ou trois) carrières de Richard Hoggart. De la fondation des cultural studies aux appropriations de la sociologie française ». Revue d'anthropologie des connaissances, 11(3) : 263-282.

DUMONT Fabienne \& SOFIO Séverine (2007). « Esquisse d'une épistémologie de la théorisation féministe en art ». Cahiers du genre, 43 : 17-43.

DuVAL Julien (2010). "Distinction studies 》. Actes de la recherche en sciences sociales, 181-182:146-156

ElIAS Norbert (1991). La Société des individus. Traduit de l'allemand par Jeanna Etoré. Paris, Fayard.

FABIANI Jean-Louis (dir.) (2001). Le Goût de l'enquête. Pour Jean-Claude Passeron. Paris, L'Harmattan.
FossÉ-PolıAK Claude (2006). Aux frontières du champ littéraire. Sociologie des écrivains amateurs. Paris, Économica.

FoucAult Michel (1973). Moi, Pierre Rivière ayant égorgé ma mère, ma sœur et mon frère... Paris, Gallimard/Julliard.

FoucAult Michel (1994). Dits et écrits, t. 1 et 2. Paris, Gallimard.

GaBORIAU Philippe (1995). Le Tour de France et le vélo. Histoire sociale d'une épopée contemporaine. Paris, L'Harmattan.

GabORIAU Philippe (2003). Les Spectacles sportifs. Grandeurs et décadences. Paris, L'Harmattan.

GinzBURG Carlo (1980) [1976]. Le Fromage et les vers. L'univers d'un meunier du $\mathrm{XV}^{e}$ siècle [Il formaggio e i vermi. II cosmo di un mugnaio del Cinquecento, Turin, Einaudi]. Traduit de l'italien par Monique Aymard. Paris, Flammarion.

Grenier Jean-Yves, Grignon Claude, Menger Pierre-Michel (dir.) (2001). Le Modèle et le récit. Paris, Maison des Sciences de l'Homme.

Grignon Claude (1996). " Le savant et le lettré, ou l'examen d'une désillusion ", Revue européenne des sciences sociales, 103 : 81-98.

GriGnon Claude (2001). " La formalisation et les sciences du récit, le cas de la sociologie ". In GRENIER Jean-Yves, GRIGNON Claude, MeNGER Pierre-Michel (dir.), Le Modèle et le récit. Paris, Maison des Sciences de l'Homme : 7-43.

Grignon Claude \& Kordon Claude (dir.) (2009). Sciences de l'homme et sciences de la nature, essais d'épistémologie comparée. Paris, Maison des Sciences de l'Homme.

GriGnOn Claude \& PASSERON Jean-Claude (1982). Sociologie de la culture et sociologie des cultures populaires. Paris, Documents du GIDES, 4

Grignon Claude \& PAsseron Jean-Claude (1985). Enquête. Cahiers du CERCOM, 1 («À propos des cultures populaires »). [En ligne] https:// enquete.reveues.org/2 [consulté le 26 juin 2017].

GriGnon Claude \& PASSERON Jean-Claude (2015) [1989]. Le Savant et le Populaire. Misérabilisme et populisme en sociologie et en littérature.

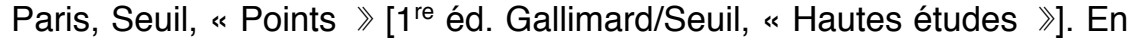
espagnol : GriGnon Claude \& PASSERON Jean-Claude (1991). Lo culto y lo popular : miserabilismo y populismo en sociologia y en literatura. Traduction de María Sonderéguer. Buenos Aires, Ediciones Nueva Vision; GriGnOn Claude \& PASSERON Jean-Claude (1992). Lo culto y lo popular : 
miserabilismo y populismo en sociologia y en literatura. Traduction de Fernando Alvarez-Uria et Julia Varela. Madrid, Las Ediciones de La Piqueta.

Grignon Claude, Collovald Annie, Pudal Bernard, Sawicki Frédéric (1991). « Un savant et le populaire. Entretien avec Claude Grignon ». Politix, $13: 35-42$

GRUEL Louis (2004). La Rébellion de 68. Une relecture sociologique. Rennes, Presses Universitaires de Rennes.

GUILLAUMIn Colette (2002) [1972]. L'Idéologie raciste. Paris, Gallimard.

HALL Stuart \& JeFFERSON Tony (dir.) (1976). Resistance through Rituals : Youth Subcultures in Post-War Britain. Londres, Hutchinson.

Hammou Karim (2008). « Penser le pouvoir avec Le Savantet le Populaire ». Journée d'étude « Le Savant et le Populaire, 1989-2008. Retour sur un débat en suspens ». SHADYC, EHESS Marseille (non publié).

HeINICH Nathalie (2000) [1991]. Etre écrivain. Paris, La Découverte.

Hoggart Richard (1970a). La Culture du pauvre. Étude sur le style de vie des classes populaires en Angleterre. Traduit de l'anglais par Françoise Garcia, Jean-Claude Garcia et Jean-Claude Passeron, présentation et index de Jean-Claude Passeron (titre original The Uses of Literacy Aspects of Working Class Life). Paris, Minuit.

HogGART Richard (1970b). Speaking to Each Other, vol. 1. Londres, Chatto et Windus.

HogGaRT Richard (2013) [1991]. 33 Newport Street. Autobiographie d'un intellectuel issu des classes populaires anglaises. Traduit de l'anglais par Christiane Grignon \& Claude Grignon avec la collaboration de Christopher Todd, présentation de Claude Grignon. Paris, Seuil, « Points »

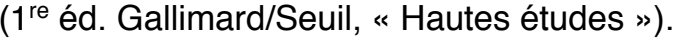

KALIFA Dominique (2005). "Les historiens français et le "populaire" ». Hermès, $42:$ 54-59.

KRÉFA Abir (2013). " La quête de l'autonomie littéraire en contexte autoritaire : le cas des écrivains tunisiens ». Sociologie, $4: 395-411$.

KRÉFA Abir (2013). Activités littéraires et rapports sociaux de sexe : le cas des écrivains tunisiens (thèse de doctorat en sociologie). Lyon, Université Lyon 2.
KRÉFA Abir (2014). "Entre injonctions à dire et à taire le corps : les voies étroites de la reconnaissance littéraire pour les écrivaines tunisiennes 》. Ethnologie française, 44(4) : 631-642.

LAGRAVE Rose-Marie (1980). Le Village romanesque. Le Paradou, Actes Sud, « Espace-temps ».

LAGRAVE Rose-Marie (1988). "Mensonge romanesque et vérité des romanciers. Une relecture du Village Romanesque ». Études Rurales, $109: 55-73$.

LAGRAVE Rose-Marie (2009). "Filiations intellectuelles et espérance sociale. Figure et œuvre de Placide Rambaud ». Études rurales, 183 51-66.

LAGRAVE Rose-Marie (2010). "Se ressaisir 》. Genre, Sexualité \& Société, $4: 1$-17. [En ligne] http://gss.revues.org

LAHIRE Bernard (2004). La Culture des individus : dissonances culturelles et distinction de soi. Paris, La Découverte.

LAHIRE Bernard (2008). La Raison scolaire. École et pratiques d'écriture, entre savoir et pouvoir. Rennes, Presses Universitaires de Rennes.

LaHIRE Bernard (2012). Monde pluriel. Penser l'unité des sciences sociales. Paris, La Découverte.

LAMONT Michèle (1995). La Morale et l'argent : les valeurs des cadres en France et aux États-Unis. Paris, Métailié.

LAMONT Michèle (2013). "En quoi Bourdieu a-t-il été utile à notre réflexion ? Le cas des États-Unis ». In CoulANGEON Philippe \& DUVAL Julien (dir.), Trente ans après La Distinction de Pierre Bourdieu. Paris, La Découverte : 59-68.

LevI Giovanni (1989). Le Pouvoir au village. Histoire d'un exorciste dans le Piémont du XVII siècle. Paris, Gallimard.

LINHART Robert (1981). L'Établi. Paris, Minuit.

MACKENZIE Caroline (2012). "Agency, un mot, un engagement ». Rives méditerranéennes, 41(1). [En ligne] http://rives.revues.org/4139 [consulté le 11 mars 2017].

MATHIEU Nicole-Claude (1991). L'Anatomie politique. Paris, Côté-Femmes. MAUgeR Gérard (1991). "Enquêter en milieu populaire 》. Genèses, 6 : 125-143. 
MAUGER Gérard (2005). " Un apprentissage tardif du métier de sociologue ». In MAUGER Gérard (dir.), Rencontres avec Pierre Bourdieu. Bellecombe-en-Bauges, Le Croquant : 239-257.

MAUGER Gérard (2006a). "Sur la violence symbolique ». In MüLLER HansPeter \& Sintomer Yves (dir.). Pierre Bourdieu, théorie et pratique. Paris, La Découverte : 84-100.

MAUGER Gérard (2006b). Les Bandes, le milieu et la bohème populaire. Études de sociologie de la déviance des jeunes des classes populaires (1975-2005). Paris, Belin.

MAUGER Gérard (2013). "Bourdieu et les classes populaires. L'ambivalence des cultures dominées ». In CoULANGEON Philippe \& DUVAL Julien (dir.), Trente ans après La Distinction de Pierre Bourdieu. Paris, La Découverte : 243-254.

MAUGER Gérard (2014). « Domination ». Le Lexique socius. [En ligne] http:// ressources-socius.info/index.php/lexique/21-lexique/154-domination [consulté le 14 janvier 2017].

MAUGER Gérard \& FossÉ Claude (1977). La Vie buissonnière. Marginalité petite-bourgeoise et marginalité populaire. Paris, Maspero.

MAUGER Gérard \& Fossé-Poliak Claude (1985). "Choix politiques et choix de recherches. Essai d'auto-socio-analyse (1973-1984) ». Cahiers " Jeunesses et Sociétés ", 3-4-5 : 27-121.

MENGER Pierre-Michel (2009). Le Travail créateur. S'accomplir dans l'incertain. Paris, Gallimard/Seuil, « Hautes études ».

MoRRISON Toni (1990). Playing in the Dark : Whiteness and The Literary Imagination. New York, Vintage Books.

MouLIN Raymonde (1971). « La Culture du pauvre. À propos du livre de Richard Hoggart ». Revue française de sociologie, 12(2) : 255-258.

MouLIN Raymonde (1992). L'Artiste, l'institution et le marché. Paris, Flammarion.

Moulin Raymonde (dir.) (1986). Sociologie de l'art. Paris, La Documentation française.

Moulin Raymonde, Passeron Jean-Claude, PAsquier Dominique, PortoVASQUEZ Fernando (1985). Les Artistes, essai de morphologie sociale. Paris, La Documentation Française.

NAUDIER Delphine \& Rollet Brigitte (2007). Genre et légitimité culturelle. Quelle reconnaissance pour les femmes ?. Paris, L'Harmattan.
NAVEL Georges (1945). Travaux. Paris, Gallimard.

Neveu Érik (1999). "Pour en finir avec l'enfantisme. Retours sur enquêtes ». Réseaux, 92-93 : 175-201.

NEveu Érik (2008). « Les voyages des cultural studies ». L'Homme, 187$188: 315-341$

NoIRIEL Gérard (1988). Le Creuset français. Histoire de l'immigration $\left(X I X^{e}-X X^{e}\right.$ siècle). Paris, Seuil.

Olivier de SARDAN Jean-Pierre (2008). La Rigueur du qualitatif. Les contraintes empiriques de l'interprétation socio-anthropologique. Louvain, Bruylant Academia.

PARETo Vilfredo (1917). Traité de sociologie générale. Traduit de l'italien par Pierre Boven. Lausanne, Payot.

PAsquali Paul \& Schwartz Olivier (2016). "La Culture du pauvre : un classique revisité. Hoggart, Les classes populaires et la mobilité sociale ». Politix, 114 : 21-45.

Pasqual Paul (2014). Passer les frontières sociales. Comment les " filières d'élite " entrouvrent leurs portes. Paris, Fayard.

PASQUIER Dominique (1989). La Culture des sentiments. L'expérience télévisuelle des adolescents. Paris, Editions de la MSH.

PASQUIER Dominique (2005). "La "culture populaire" à l'épreuve des débats sociologiques ». Hermès, $42:$ 60-69.

PASSERON Jean-Claude (2006) [1991]. Le Raisonnement sociologique. Un espace non poppérien de l'argumentation. Paris, Albin Michel, nouvelle édition revue et augmentée (première édition Paris, Nathan).

Passeron Jean-Claude \& Pedler Emmanuel (1991). Le Temps donné aux tableaux. Compte rendu d'une enquête au musée Granet. Marseille, Imerec.

PASSERON Jean-Claude \& GRUMBACH Michel (dir.) (1985). L'CEil à la page : enquête sur les images et les bibliothèques. Paris, Bibliothèque publique d'information, Service des études et de la recherche.

PAsSERON Jean-Claude (dir.) (1999). Richard Hoggart en France. Paris, Bibliothèque publique d'information.

Pedler Emmanuel (2010). « Les sociologies de la musique de Max Weber et Georg Simmel. Une théorie relationnelle des pratiques musiciennes ». L'Année sociologique, 60(2) : 305-330. 
Pedler Emmanuel (2016). L'Esprit des lieux. Paris, Éditions de l'EHESS. Pedler Emmanuel \& Bourbonnaud David (2002a). " L'offre du festival "in" d'Avignon : effet d'imposition ou stimulation culturelle ? ". Actes du colloque d'Amiens.

Pedler Emmanuel \& Bourbonnaud David (2002b). « La programmation du Festival. Un pacte de confiance entre l'organisation festivalière et son public ». In EtHIS Emmanuel (dir.), Avignon : le public réinventé. Paris, La Documentation française : 131-159.

PedLeR Emmanuel \& ZerbiB Olivier (2001). Les Nouvelles Technologies à l'épreuve des bibliothèques. Paris, Bibliothèque publique d'information. Peterson Richard A. \& Simkus Albert (1992). " How Musical Tastes Mark Occupational Status Groups ». In LAMONT Michèle \& FouRNIER Marcel, Cultivating differences : Symbolic Boundaries and the Making of Inequality. Chicago, The University of Chicago Press : 152-168.

Peterson Richard A. (1992). " Understanding audience segmentation : From elite and mass to omnivore and univore ». Poetics, 21(4) : 243-258.

Peterson Richard A. (1997). "The Rise and Fall of Highbrow Snobbery as a Status Marker ». Poetics, 25(2-3) : 75-92.

Peterson Richard A. \& Kern Roger M. (1996). "Changing Highbrow Taste : from Snob to Omnivore ». American Sociological Review, 61(5) : 900-907.

PUDAL Bernard (1991). "Le populaire à l'encan ». Politix, 13 : 53-64.

RANCIÉRE Jacques (1981). La Nuit des prolétaires. Archives du rêve ouvrier. Paris, Fayard.

RANCIÈRE Jacques (2011). La Leçon d'Althusser. Paris, La Fabrique.

Revel Jacques (dir.) (1996). Jeux d'échelles. La micro-analyse à l'expérience. Paris, Gallimard/Seuil.

Robinson Eric E. (1968). The New Polytechnics. The People's Universities. Harmondsworth, Penguin.

RoEDIGER David (1991). The Wages of Whiteness : Race and the Making of the American Working Class. New York, Verso.

SAPIRO Gisèle (2013a). «La carrière internationale de La Distinction ». In COULANGEON Philippe \& DUVAL Julien (dir.), Trente ans après La Distinction de Pierre Bourdieu. Paris, La Découverte : 45-58.
SAPIRO Gisèle (2013b). " Le champ est-il national ? La théorie de la différenciation sociale au prisme de l'histoire globale ". Actes de la recherche en sciences sociales, $200: 70-85$.

SCHILLING Heinz (1981). Konfessionskonflikt und Staatsbildung. Eine Fallstudie über das Verhältnis von religiösem und sozialem Wandel in der Früh Neuzeit am Beispiel der Grafschaft Lippe. Gütersloh, Bertelsmann. ScotT James (2008). La Domination et les arts de la résistance. Fragments du discours subalterne. Traduction française par Olivier Ruchet. Paris, Editions Amsterdam.

Servien Pius (1935). Principes d'esthétique. Problèmes d'art et langage des sciences. Paris, Boivin.

SINGLY François (DE) (1998). "Bourdieu : nom propre d'une entreprise collective ». Le Magazine littéraire, $369:$ 39-44.

SUTTON-SMITH Brian (1970). "Psychology of Childlore : The Triviality Barrier ». Western Folklore, 29(1) : 1-8.

TAINE Hyppolite (1858). Essais de critique et d'histoire. Paris, Hachette.

VEYNE Paul (1971). Comment on écrit l'histoire. Essai d'épistémologie. Paris, Seuil.

VEYNE Paul (2008). Foucault. Sa pensée, sa personne. Paris, Albin Michel. Weber Max (1998) [1921]. Sociologie de la musique. Les fondements rationnels et sociaux de la musique [Die rationalen und soziologischen Grundlagen der Musik, Tübingen, Drei Masken Verlag]. Traduit de l'allemand par Jean Molino et Emmanuel Pedler. Paris, Métailié.

Wimmer Andreas \& Glick SCHILleR Nina (2002). « Methodological Nationalism and Beyond : Nation-State Building, Migration and the Social Sciences ». Global Network, 2 : 301-334. 